Facilitating Communication: How to Truly Understand What Patients Mean

By: Shattell, M., \& Hogan, B.

Shattell, M., \& Hogan, B. (2005). Facilitating communication: How to truly understand what patients mean. Journal of Psychosocial Nursing and Mental Health Services, 43(10), 29-32.

Made available courtesy of Slack Inc.: http://www.slackinc.com/

*** Note: Figures may be missing from this format of the document

**** Note: Slack Inc. requests that interested viewers (Scholars and Researchers) understand that these articles can be accessed for their personal and reference use. The articles should not be reproduced in large quantities for distribution in classrooms or seminars without obtaining the rights from Slack Incorporated. We provide licensed reprints for distribution purposes for an appropriate fee.

Article:

"Wisdom begins with the definition of terms." Socrates

The nurse-patient relationship will always remain pivotal to effective management of illness. Peplau (1952/1991) maintained that understanding was an essential component of the nurse-patient relationship. Consistent with Peplau's assertion, Cleary, Edwards, and Meehan (1999) found that understanding significantly influenced nurse-patient interactions in acute psychiatric-mental health settings. Jackson and Stevenson (2000) cited understanding as a central theme in their study about the reasons people with acute psychiatric illnesses need nurses. Although understanding is what patients want, studies suggest they do not experience it from nurses (Shattell, 2002; Thomas, Shattell, \& Martin, 2002). In fact, one study revealed that the greatest understanding came from other patients (Thomas et al., 2002). In this article, we describe communication guidelines useful to nurses in facilitating patients' experience of being understood.

\title{
THE PROBLEM OF MISCOMMUNICATION
}

Culture provides a prototype for examining the skills necessary to facilitate understanding. A focus on cultural sensitivity and diversity training has raised awareness of the importance of clarifying individual meanings to facilitate understanding with patients who are clearly different. Even when nurses are acutely aware of possible differences in meanings, opportunities for misunderstanding and miscommunication abound. For example, in a study of non-Aboriginal health care providers and Aboriginal patients, Cass et al. (2002) found that health care providers did not even recognize their errors in understanding. Obviously, shared meanings of concepts is requisite to understanding (Cass et al., 2002).

Shared meanings of concepts are particularly difficult when patients and providers speak different languages. In a study about the use of nurse-interpreters with Spanish-speaking patients, Elderkin-Thompson, Silver, and Waitzkinc (2001) found that approximately half of interpreter-mediated encounters had serious miscommunication problems affecting either the health care provider's understanding of symptoms or the credibility of the patient's concerns. In addition, Maltby (1999) found that interpreters often edit out important aspects of patient communication, clarifying that interpreting is "more than moving words from one language to another" (p. 250).

People tend to think culture or diversity as concepts pertaining only to people from other countries or people who are clearly different (Evans \& Severston, 2001). However, even within one culture, tremendous diversity exists. Canales (1997) indicated that one must avoid stereotyping based on cultures and recognize individual differences within cultures.

Misunderstandings can com-promise quality of care. An example from our clinical practice involved a foreignborn physician who asked a patient on an inpatient psychiatric unit, "How did you sleep last night?" The patient 
replied, "I slept like a log." The physician thought the patient was delusional (i.e., the patient thinks he is a log) and increased the patient's antipsychotic medications. The physician, whose first language was not English, misinterpreted the patient's response to his question, and rather than seeking clarification, accepted a concrete under-standing of the phrase "slept like a log," which resulted in treatment changes.

Another example involved a newly admitted patient on an inpatient psychiatric unit who had locked himself in the bathroom while yelling, "It's coming down. It's coming down!" Because the patient was known to experience religious delusions, the nurses assumed the patient was talking about Jesus and the second coming of Christ, and administered several injections of haloperidol. After further inquiry (i.e., clarification of meaning), it became clear the patient was referring to very large, strangulated hemorrhoids, which subsequently required emergency surgical intervention.

These examples highlight the need for nurses and other health care providers to better understand their patients with mental illnesses. How can this be done? We can begin by returning to the essence of psychosocial nursing.

\section{THE ESSENCE OF PSYCHOSOCIAL NURSING}

Nurses in all roles try to elicit symptom descriptions from patients during health care encounters (American Nurses Association [ANA], 1995). To effectively manage symptoms, nurses must listen and attend to patients' perceptions of their experiences (Haworth \& Dluhy, 2001). In a study by Evans and Severston (2001), listening to patients' stories increased the sensitivity of nursing students to the diversity of all individuals involved. In this innovative learning approach, students were taught to be truly sensitive to individual meanings by allowing patients to tell their whole story without judgment and seeking only to clarify and validate what was meant (Evans \& Severston, 2001). In addition, Bailey and Tilley (2002) indicated that patients' stories allow health care providers to access their reality and their truth, which is essential to providing appropriate care. Specific c guidelines nurses can use to facilitate an understanding of what patients mean are discussed in the following section.

\section{SPECIFIC COMMUNICATION GUIDELINES}

Understanding can be facilitated by one overarching communication principle: Nurses may not comprehend what patients mean. In the study by Elderkin-Thompson et al. (2001), the most successful translations by nurseinterpreters occurred when physicians and nurse-interpreters worked slowly to understand the patient's opening comments and verified each fact. The only way to understand patients' experiences is to:

- Be aware of taken-for-granted meanings.

- Commit to making the time to seek clarification and explore meanings.

- Restate what was heard to ensure understanding and demonstrate active listening.

- Ask open-ended questions that begin with requests, such as "Tell me more about that" or "What was that like?"

- Frequently and regularly check for understanding by saying, "Let me see if I understood correctly."

Some communication styles block understanding. For ex-ample, open-ended questions are often used in psychiatric-mental health nursing, but questions that begin with the word "why" are not as helpful as those that begin with phrases such as, "Tell me more about that" or "What was that like?" "Why" questions take patients out of their descriptions of their current experiences or feelings and channel them into either an intellectual or defensive response.

Comments that convey "complete understanding" also block understanding because it is impossible to completely understand another's experience. According to Jackson and Stevenson (2000): 
a nurse who has not experienced mental illness personally cannot know what it feels like. Even if they themselves have experienced mental health problems, they still cannot know what it feels like for that particular client. (p. 382)

Nurses should avoid using the phrase "I completely understand." Instead, phrases such as "Let me see if I understood correctly" are more appropriate. Seeking clarification to help patients elucidate meanings helps deepen under-standing, but clarification is never absolute. Nurses need to "be guided by the [patient's] definition of what is needed for her/him" (Jackson \& Stevenson, 2000, p. 382). Communication principles such as those in this article may improve nurses' chance of under-standing patients through meaningful therapeutic relationships.

Through the formation and maintenance of therapeutic relationships, and through repeated clarification and validation of patients' experiences and, thus, meaning, nurses are able to discern the nuances of patient communication (Bailey \& Tilley, 2002). According to the ANA's (1995) definition, nursing requires the: integration of objective data with knowledge gained from an understanding of the patient['s]... subjective experience ... and [for] provision of a caring relationship that facilitates health and healing. (p. 6)

\section{CONCLUSION}

All nurse-patient interactions occur within the context

of many influencing variables, such as gender, ethnicity, culture, expectations, and illness. The degree to which nurses embrace a holistic, egalitarian view of their patients may determine the degree to which individual meanings are sought. Thus, the way to ascertain patients' reality is through clarification and validation of meaning (Haworth \& Dluhy, 2001).

Patients long for greater understanding within the context of the therapeutic nurse-patient relationship. Being aware of taken-for-granted meanings and clarifying all communication can improve nursing practice. Patients experience greater satisfaction with nursing care when they are truly understood. Hopefully, the communication guidelines offered in this article can begin to close the gap between patients feeling misunderstood and patients saying, "that nurse truly understood me."

\section{KEYPOINTS}

1. Patients experience greater satisfaction with nursing care when they are truly understood.

2. Being aware of taken-for-granted meanings and committing the time to seek clarification and explore meanings are two basic principles that can facilitate understanding.

3. Techniques nurses can use to facilitate understanding include restating what was heard to ensure understanding and to demonstrate active listening, asking open-ended questions, and checking for understanding.

Do you agree with this article? Disagree? Have a comment or questions? Send an e-mail to Karen Stanwood, Executive Editor, at kstanwood@slackinc.com. We're waiting to hear from you!

\section{REFERENCES}

American Nurses Association. (1995). Nursing's social policy statement. Washington, DC: Author.

Bailey, P.H., \& Tilley, S. (2002). Storytelling and the interpretation of meaning in qualitative research. Journal of Advanced Nursing, 38, 5 74-583.

Canales, M. (1997). Narrative interaction: Creating a space for therapeutic communication. Issues in Mental Health Nursing, 18, 477-494. 
Cass, A., Lowell, A., Christie, M., Snelling, P., Flack, M., Marrnganyin, B., et al. (2002). Sharing the true stories: Improving communication between Aboriginal patients and healthcare workers. The Medical Journal of Australia, 176, 466-470. Retrieved July 12, 2004, from http://www.mja.com. au/public/issues/176_10_200502/ cas10830_fm.html

Cleary, M., Edwards, C., \& Meehan, T. (1999). Factors influencing nurse-patient interaction in the acute psychiatric setting: An exploratory investigation. Australian and New Zealand Journal of Mental Health Nursing, 8, 109-116.

Elderkin-Thompson, V., Silver, R., \& Waitzkinc, H. (2001). When nurses double as interpreters: A study of Spanish-speaking patients in a U.S. primary care setting. Social Science \& Medicine, 52, 1343-1358.

Evans, B.C., \& Severston, B.M. (2001). Storytelling as cultural assessment. Nursing \& Healthcare Perspectives, $22,180-183$.

Haworth, S., \& Dluhy, N. (2001). Holistic symptom management: Modeling the interaction phase. Journal of Advanced Nursing, 36, 302-3 10.

Jackson, S., \& Stevenson, C. (2000). What do people need psychiatric and mental health nurses for? Journal of Advanced Nursing, 31, 3 78-3 88.

Maltby, H.J. (1999). Interpreters: A double-edged sword in nursing practice. Journal of Transcultural Nursing, 10, 248-254.

Peplau, H. (1991). Interpersonal relations in nursing: A conceptual frame of reference for psychodynamic nursing. New York: Springer. (Original work published 1952)

Shattell, M. (2002). "Eventually it'll be over": The dialectic between confinement and freedom in the phenomenal world of the hospitalized patient. In S. Thomas \& H. Pollio (Eds.), Listening to patients: A phenomenological approach to nursing research and practice (pp. 214-236). New York: Springer.

Thomas, S., Shattell, M., \& Martin, T. (2002). What's therapeutic about the therapeutic milieu? Archives of Psychiatric Nursing, 16, 99-107. 